EPiC Series in Computing
Volume 66, 2019, Pages 60-67
Proceedings of 6th International OM-
NeT++ Community Summit 2019

\title{
Towards a Bidirectional Coupling of Pedestrian Dynamics and Mobile Communication Simulation *
}

\author{
Stefan Schuhbäck ${ }^{1}$, Nico Daßler ${ }^{1}$, Lars Wischhof ${ }^{1}$, and Gerta Köster ${ }^{1}$ \\ Munich University of Applied Sciences, Munich, Germany \\ stefan. schuhbaeck| dassler | wischhof | koester@hm.edu
}

\begin{abstract}
In order to accurately evaluate new concepts and protocols for mobile communication networks, realistic mobility models are needed. Furthermore, for use cases which have a bidirectional dependency between communication and mobility, changes in communication lead to changes in mobility and vice versa, thus requiring an online coupling between models. Therefore, bidirectional coupling to incorporate realistic mobility patterns is state of the art in the analysis of Vehicular Ad-Hoc Network (VANET) applications.

However, the same need exist for use cases where the mobile users are pedestrians rather than vehicles. Therefore, this paper introduces our current, on-going work on connecting $\mathrm{OMNeT}++$ and Vadere, an open source simulation framework for microscopic pedestrian dynamics, to benefit from state of the art pedestrian mobility models in mobile communication use cases. The presented coupling is based on the existing Traffic Control Interface (TraCI) protocol used in the Veins (Vehicles in Network Simulation) framework to connect OMNeT++ with SUMO.
\end{abstract}

\section{Introduction}

When designing communication technologies and protocols, network simulation is a widely used method in order to evaluate their performance. Pedestrians are a major user group of mobile communication systems such as cellular communication systems (e.g. LTE, LTE-A, 5G), Wireless Local Area Networks (WLAN), Bluetooth and many more. While typical tools for network simulation used in the research community support rather simplified mobility models for pedestrians, researchers in the field of pedestrian dynamics are developing and applying accurate locomotion models in specialized pedestrian simulation frameworks. Therefore, this paper investigates different approaches for coupling network and pedestrian simulation.

Application areas include Intelligent Transportation Systems (ITS) which increase the capacity and efficiency of (multi-modal) transportation systems and the underlying infrastructure

\footnotetext{
*We thank the research office (FORWIN) of the Munich University of Applied Sciences for supporting the research collaboration. The authors gratefully acknowledge the support by the Faculty Graduate Center CeDoSIA of TUM Graduate School at Technische Universität München, Germany. The authors also acknowledge the financial support by the Federal Ministry of Education and Research of Germany in the framework of roVer (project number 13FH669IX6).
} 


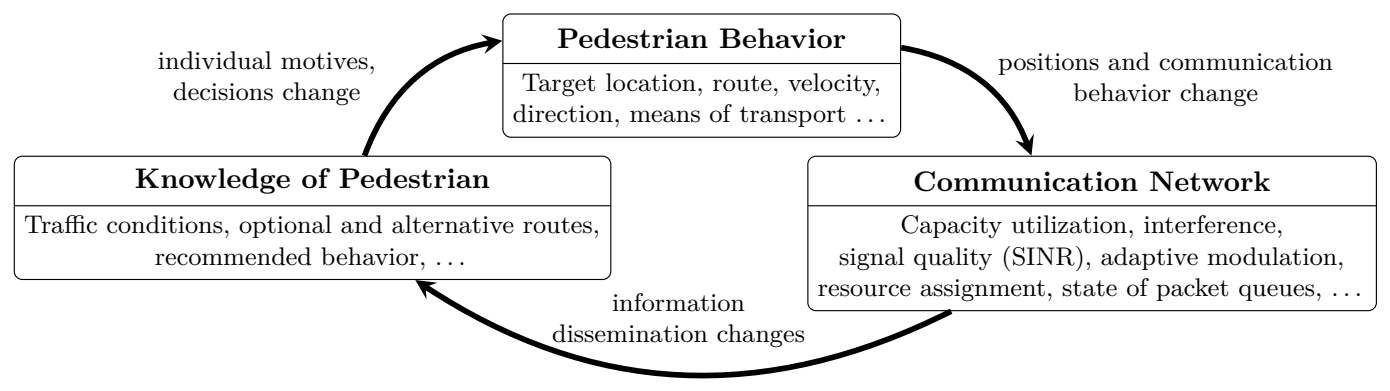

Figure 1: Feedback cycle: mobility, communication network, knowledge.

by disseminating mobility-related information. Use cases such as distributing information concerning public transportation mostly address pedestrians. In order to analyze communication in these use cases, a realistic mobility model for pedestrians is needed. Furthermore, information received by a pedestrian can lead to a change in the mobility behavior which will affect the communication network, leading to a feedback cycle as illustrated in Fig. 1.

In general, a possible solution would be either to extend an existing network simulator with highly accurate locomotion models or to extend an existing pedestrian/crowd simulation tool with a detailed communication simulation. However, because of the high implementation effort required for these extensions, this paper focusses on a coupling of existing pedestrian mobility simulators with a network simulator - an approach that has been successfully implemented for the simulation of VANETs by including vehicle movement patterns simulated in a vehicular traffic simulator.

Contributions The main contributions of this paper are the following: (i) a brief evaluation of possible options for coupling pedestrian mobility and network simulation - ranging from an unidirectional, trace-based coupling to a bidirectional online-coupling of both simulators; (ii) an extension of the well-known Veins [11] framework that couples the vehicular traffic simulator SUMO [9] with network simulations based on OMNeT++ to support receiving pedestrian mobility information; (iii) a novel interconnection of OMNeT++ and Vadere [5], an open source simulation framework for microscopic pedestrian dynamics. The latter two are also made available as part of a public open-source project, in order to enable further research. ${ }^{1}$

\section{Related Work}

In this section a short introduction to mobility models incorporated in OMNeT++ is given, followed by an existing bidirectional coupling of vehicular mobility and network simulators. The section closes with a brief overview of current mobility models from pedestrian dynamics and the Vadere simulation framework.

\subsection{Mobility Models in the INET Framework}

The INET ${ }^{2}$ Framework for OMNeT++ is a model suite for the simulation of wired, wireless and mobile networks. It includes the notion of a generic mobility interface, which connects communi-

\footnotetext{
${ }_{1}$ https://github.com/roVer-HM/veins/tree/omnet-summit-2019 Sub-project: vader_traci_demo

${ }^{2}$ https://inet.omnetpp.org/
} 
Table 1: A selection of existing node mobility models incorporated in the INET Framework [4].

\begin{tabular}{|c|c|c|c|}
\hline Stationary & Deterministic & Stochastic & Trace-Based \\
\hline $\begin{array}{l}\text { Place nodes at start } \\
\text { but no movement during } \\
\text { simulation. }\end{array}$ & $\begin{array}{l}\text { Mobility defined through } \\
\text { deterministic processes. }\end{array}$ & $\begin{array}{l}\text { Mobility defined with a } \\
\text { configurable random ele- } \\
\text { ment. }\end{array}$ & $\begin{array}{l}\text { Trace files generated } \\
\text { from external mobility } \\
\text { providers or experimen- }\end{array}$ \\
\hline $\begin{array}{l}\text { i.e. StationaryMobility, } \\
\text { StaticGridMobility }\end{array}$ & $\begin{array}{l}\text { i.e. LinearMobility, } \\
\text { CircleMobility, Tractor- } \\
\text { Mobility }\end{array}$ & $\begin{array}{l}\text { i.e. Random Waypoint- } \\
\text { Mobility, GaussMarkov- } \\
\text { Mobility, MassMobility }\end{array}$ & $\begin{array}{lr}\begin{array}{lr}\text { i.e. } & \text { BonnMotion- } \\
\text { Mobility, } & \text { Ns2Motion- } \\
\text { Mobility, } & \text { AnsimMobility }\end{array}\end{array}$ \\
\hline
\end{tabular}

cation nodes to a location in the three dimensional space. Depending on the implementation of the interface, different mobility models are possible. Table 1 shows the classification of mobility models within the INET Framework. The main groups are stationary, deterministic, stochastic and trace-based. Stationary models simply connect a node to a fixed location without moving the node during the simulation. This approach is useful, for instance, to place cellular towers or road side units (RSU) during simulation initialization. Deterministic and stochastic models update the location of the communication node based on deterministic or stochastic algorithms such as the CircleMobilty model, which moves a node along the circumference of a circle, or the Random WaypointMobiliy model where nodes move with a random speed along linear paths, constructed based on the current location and a new target location selected at random [4].

Lastly, trace-based mobility providers supply the position of mobile nodes based on prerecorded trajectory traces. For instance, the BonnMotionMobility provider utilizes the trace format of BonnMotion, a mobility scenario generation and analysis tool [1].

However, all incorporated models show shortcomings to the aforementioned requirements of accurately modelling pedestrian movement and allowing a bidirectional feedback between communication and mobility models. The existing deterministic and stochastic models do not accurately model pedestrian movement. While trace-based providers can include accurate movement, by extracting traces from dedicated pedestrian dynamics simulators such as Vadere, the use of pre-generated traces does not allow interaction, which makes them impractical for use cases containing feedback cycles as illustrated in Fig. 1.

The focus of this paper lies in the coupling of microscopic pedestrian mobility models, thus macroscopic mobility model integrated in OMNeT ++ as discussed in [8] are out of scope for this contribution.

\subsection{Coupling of Vehicular Mobility and Network Simulation}

The challenge to incorporate realistic, interactive mobility models is already known from use cases where communication in VANET applications is analyzed. Therefore, the Veins (Vehicles in Network Simulation) framework couples OMNeT++ with SUMO (Simulation of Urban MObility), as an external mobility provider, to overcome the previously discussed shortcomings. [11]

SUMO is a microscopic traffic simulator operating on continuous space and discrete time steps. The main microscopic model used is the car following model in which the speed of a given car is determined by its current speed, distance to the leading car as well as the speed of the leading car [7]. SUMO also includes tools needed to create the simulation topography such as road networks, footpaths, traffic lights and intersections. It therefore allows the simulation of a variety of use cases ranging from vehicular to public transportation and pedestrian mobility [9].

The bidirectional coupling introduced by Veins is implemented by extending both simulators 
with a communication interface, which allows the exchange of commands between both domains. Each simulator holds its own simulation time. The event driven nature of OMNeT++ is used to schedule sim_step commands, which are used to synchronize the simulation loops. When OMNeT++ triggers a sim_step command, SUMO will advance to the given simulation time and returns the updated position to $\mathrm{OMNeT}++$ which in turn uses that information to update positions, add new nodes or remove nodes which are no longer part of the simulation. Subsequently, $\mathrm{OMNeT}++$ simulates the communication protocol and relays the result back to SUMO to trigger changes in the road traffic simulation such as speed adjustment or lane changes based on the simulated VANET application [11].

Technically, the coupling is based on the Traffic Control Interface (TraCI) over a TCP connection. TraCI was introduced by [12] to create a generic protocol to exchange mobility primitives between road traffic and network simulators. Later on, TraCI was extended with control, value retrieval, state change and subscription commands. Control commands handle simulation initialization, configuration as well as advancing the simulation to the next synchronization point (sim_step). Value retrieval, state change and subscription commands are implemented for common objects available in SUMO such as vehicle, person and route. Each subscribed variable will be part of the sim_step response SUMO sends to OMNeT++. [2]

\subsection{Pedestrian Dynamics and the Vadere Framework}

To describe microscopic pedestrian movements, many different modeling approaches exist such as cellular automata (CA) [13], social forces model (SFM) [3] or the optimal steps model (OSM) [10]. A CA represents the topography as cells, which are either empty, containing a pedestrian or an obstacle, which renders the cell unusable for pedestrians. The pedestrians find the target, moving from cell to cell, using some sort of floor field which defines the closeness to the expected target. In social force models, pedestrians move in continuous space where "social" forces act on the pedestrian by attracting it to the target whilst obstacles and other pedestrian repel the pedestrian to prevent overlapping and collisions. OSM also uses continuous space but models the movement of pedestrians based on a series of discrete steps. Each step is chosen by finding the optimal position within the step length radius. The optimization also uses a floor field to find the optimal position taking into account the geodesic distance to the target as well as repulsion effects of neighboring pedestrians and obstacles [5].

All these approaches have advantages and disadvantages depending on the specific use case to be investigated. Therefore, the models need to be compared to each other and experimental data. The crowd simulation framework Vadere ${ }^{3}$ has a generic approach which allows the implementation of different microscopic locomotion models. The models can be compared visually using the graphical user interface (GUI) of Vadere or by comparing simulation results extracted by an array of extensible data processors that collect a variety of measurements such as evacuation time, density data or overlap count [5].

Regarding the requirements identified in Sec. 2.1 - accurate mobility models and bidirectional coupling with network simulation - Vadere provides a possible solution. The access to different pedestrian mobility models well known in the pedestrian dynamics field satisfies the first requirement. Due to similar handling of the simulation loop in Vadere, SUMO and OMNeT++, a coupling is possible by extending Vadere with a TraCI interface.

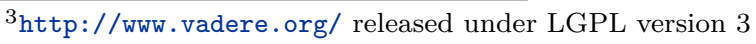




\section{Coupling Pedestrian and Communication Simulation}

As outlined in Sec. 1, two options for coupling pedestrian and network simulation have been implemented: (i) coupling with the traffic simulator SUMO; (ii) coupling with the crowd simulator Vadere. These are described in the following two subsections.

\subsection{Extending Veins to Support Pedestrians Modeled in SUMO}

TraCI offers different APIs to retrieve and change the state of simulated objects (e.g. junctions, vechicles). Furthermore, it allows to subscribe to the values of these APIs which will cause the values to be returned when the sim_step command is called (see Sec. 2.2). Veins primarily uses the vehicle, junction and simulation APIs. As pedestrians are not treated as vehicles in SUMO, the mechanism used by Veins will not cause the automatic retrieval of pedestrian variables and positions.

Therefore, the following extensions were implemented in Veins to support pedestrian value retrieval: The process of subscribing to pedestrians is quite similar to the process of subscribing to vehicles. Firstly, it is necessary to get a list containing the IDs of all active pedestrians. This can be done by subscribing to the list or by explicitly requesting it. In general, the subscription is primarily used while the explicit request can act as a backup mechanism in case the subscription does not contain the expected information. Secondly, each ID is used to subscribe to some variables (position, road id, speed, angle and type) of that specific pedestrian. Lastly, the subscribed data is used to create/remove pedestrians and to update their position and status in OMNeT++. The last step is repeated for each sim_step.

While implementing the retrieval of pedestrian mobility information in Veins, the module which manages subscriptions via TraCI were restructured: The primary focus was to ease the implementation when adding new subscriptions (e.g. a subscription for pedestrians) and to increase readability. The changes feature a simple class hierarchy which uses a different manager for each TraCI API at the lowest level. These managers are capable of monitoring their subscription result and provide an API to return the information in a simple way.

\subsection{Bidirectional Coupling of Vadere and OMNeT ++}

Motivated by the assumption that a bidirectional coupling of Vadere and OMNeT++ via TraCI is feasible (Sec. 2.3), we focus on a proof-of-concept implementation of a TraCI server in Vadere analogous to the approach for SUMO (Sec. 3.1). Whenever possible, it uses the same mechanisms and parameter names, allowing a simple replacement of SUMO with Vadere for simulations with pedestrian mobility.

In addition, the configuration in the startup-phase of the simulation was extended for Vadere: Instead of sending a XML-based configuration containing the location of needed input files, the newly added ScenarioManagerVadere streams the scenario description directly to the Vadere server instance. It is also possible to send previously calculated (i.e. cached) floor fields to reduce initialization time if the topography did not change. These steps are illustrated in the state machine in Fig. 2. When the scenario is loaded, Vadere starts the simulation, waits at $t=0 s$ and prepares for upcoming commands in the Wait for command state.

The sequence diagram on the right-hand side of Fig. 2 shows how the subscription manager interacts with Vadere. Veins subscribes to the person id list variable, which contains a list of string based identifiers for pedestrians currently in the simulation. For each returned identifier not already known, a new subscription is created, which contains the following person variables: position2D, speed, edge, angle, typeID. At time of writing, Vadere returns default values for 


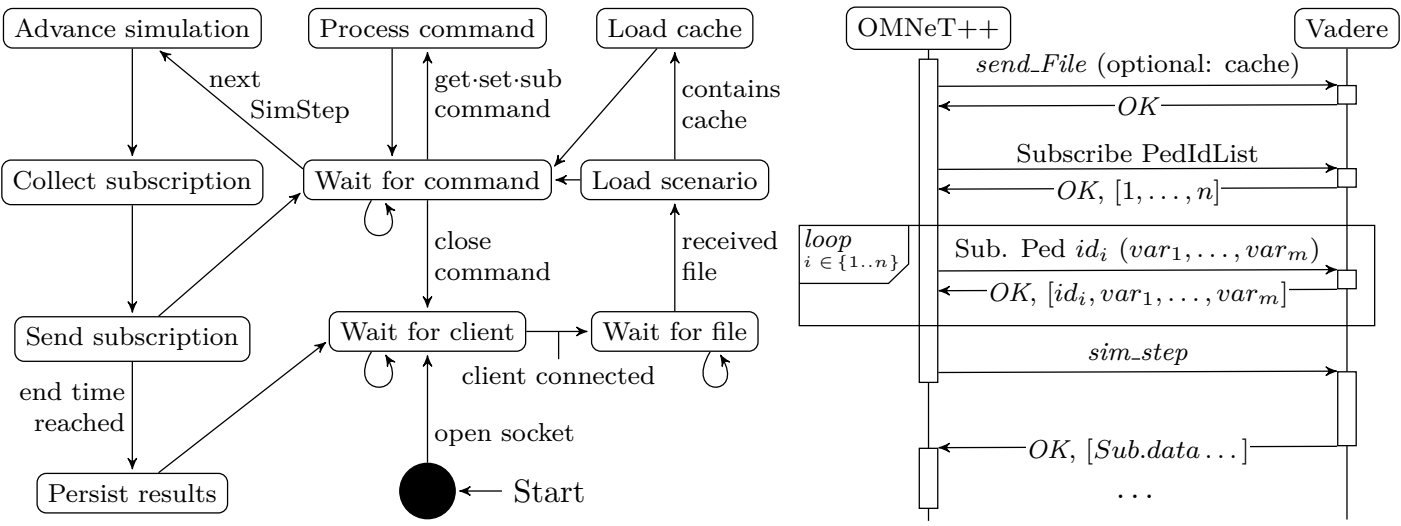

Figure 2: State machine and sequence diagram for Vadere as TraCI server.

edge and angle, because these values have no meaning in the context of Vadere. The typeID is set to "pedestrian" to inform OMNeT++ about the node type the pedestrian should be connected to. With each execution of the sim_step command, Vadere advances the simulation to the received simulation time and returns the previously subscribed data. The subscription manager ensures that newly added pedestrians are connected to the correct communication node and new subscriptions are created.

Furthermore, extensions to the Person API are necessary to address the differences between the person and pedestrian model of SUMO and Vadere. One key difference is the path selection, where in SUMO a list of stages (walk, ride, stop) describes the pedestrians movement through the scenario. The walking stage for instance contains an edge along which the pedestrian moves at a constant speed [6]. These elements are part of the Person API with corresponding methods such as getNextEdge or appendWalkingStage. The path finding in Vadere, however, is influenced by a target list. Based on the next target, first element in the list, Vadere transparently delegates the target location to the currently active locomotion model, which uses this information, among others, to calculate the next position. In the case of the optimal steps model, the correct floor field is selected to calculate the next optimal step for the given pedestrian, taking also into account obstacle repulsion and neighboring pedestrians. At time of writing, all meaningful Person API retrieval methods, such as getID or getPostion2D are implemented, retrieving the corresponding pedestrian attributes. Furthermore, a subset of the Polygon API is implemented to transfer obstacle information. Additionally, new API methods are added to allow the retrieval and change of the target list to modify pedestrian behavior based on events generated by $\mathrm{OMNeT}++$.

A simple test scenario is used to confirm that the coupling works as expected. The test scenario consist of two sources which create pedestrians at a constant rate. Each source has a corresponding target such that each created pedestrian has this target in its target list. A simplified communication application will inform selected pedestrian that target-1 (i.e. a subway entrance) is overcrowded and that target-2 should be used instead. For this test case, only five pedestrians are informed at pre-defined points in time.

Fig. 3 shows the topography of the simulation at time $t=263.2 \mathrm{~s}$, both in the OMNeT++ graphical user interface (3a) as well as in online visualization provided by Vadere (3b). Pedestrian 169 received the change event three time steps earlier at $t_{-3}=262.0 \mathrm{~s}$. Fig. 3b additionally plots the trajectories of previous pedestrians, marking the way normally used without receiv- 


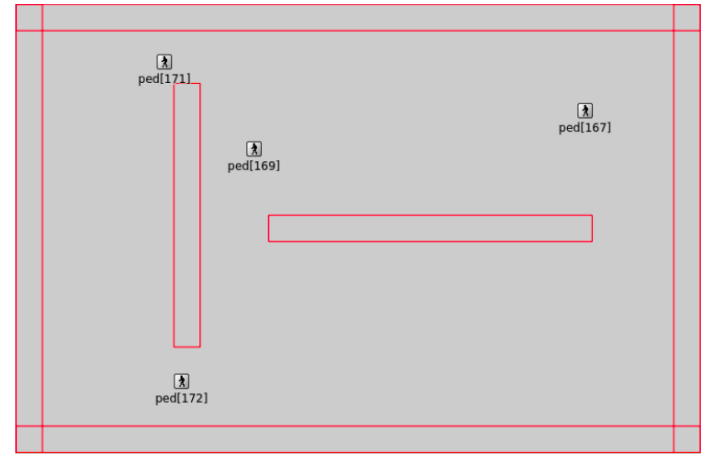

(a) OMNeT ++ with world view loaded from Vadere.

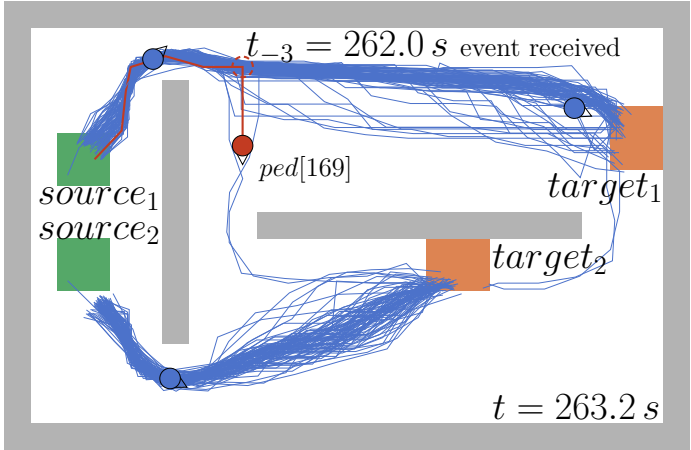

(b) Vadere topography with trajectory traces.

Figure 3: Example scenario where some pedestrians receive a signal from $\mathrm{OMNeT}++$ to change their respective target.

ing a new target. The red trajectory of pedestrian 169 clearly follows the normal path until $t_{-3}$ is reached and target_2 is new target. During the simulation setup, OMNeT++ uses the polygon API to retrieve information about the scenario layout. The red polygons on the left represent the walls (obstacles) defined in the Vadere scenario description. At time of writing, other topography elements such as sources and targets are not yet connected to the polygon API.

This shows that the coupling indeed allows a fine grained access to the mobility model. Note that the TraCI implementation only accesses generic Vadere objects, thus allowing a transparent replacement of the underling locomotion model without changing the communication simulation running in $\mathrm{OMNeT}++$.

\section{Conclusion}

In this paper, we present our current, on-going work on connecting state-of-the-art pedestrian mobility models with $\mathrm{OMNeT}++$ to allow the analysis of use cases containing feedback cycles between communication and node mobility. The extension added in Veins allows access to the pedestrian model contained in the widely-used SUMO simulation framework. Secondly, with the connection of Vadere and $\mathrm{OMNeT}++$, using the existing TraCI interface, we want to foster reuse and simplify the adoption of well known pedestrian locomotion models in communicationcentric use cases. 


\section{References}

[1] Nils Aschenbruck et al. "BonnMotion: a mobility scenario generation and analysis tool". In: Proceedings of the 3rd International ICST Conference on Simulation Tools and Techniques. ICST, 2010. DOI: 10.4108/icst.simutools2010.8684.

[2] DLR. Simulation of Urban Mobility (SUMO). 2019. URL: https://sumo.dlr.de/wiki/ TraCI.

[3] Dirk Helbing and Péter Molnár. "Social Force Model for pedestrian dynamics". In: Physical Review E 51.5 (1995), pp. 4282-4286. DOI: 10.1103/PhysRevE.51.4282.

[4] INET. INET Framework - Open-Source OMNeT++ Model Suite for Wired, Wireless and Mobile Networks. 2019. URL: https://inet . omnetpp .org/docs/users-guide/chmobility.html (visited on 07/11/2019).

[5] Benedikt Kleinmeier et al. "Vadere: An Open-Source Simulation Framework to Promote Interdisciplinary Understanding". In: accepted to be published in Collective Dynamics (2019).

[6] Daniel Krajzewicz et al. "Including Pedestrian and Bicycle Traffic into the Traffic Simulation SUMO". In: 10th ITS European Congress. 2014. URL: https://elib.dlr.de/ 90621/.

[7] Daniel Krajzewicz et al. "Recent development and applications of SUMO-Simulation of Urban MObility". In: International Journal On Advances in Systems and Measurements 5.3844. 2012.

[8] Vishnupriya Kuppusamy et al. "Community-based Mobility Model and Probabilistic ORBIT Mobility Model Implementations in OMNeT++". In: Proceedings of the 5th International OMNeT++ Community Summit. EasyChair, Oct. 23, 2018. DOI: 10.29007/4tv9.

[9] Pablo Alvarez Lopez et al. "Microscopic Traffic Simulation using SUMO". In: 2018 21st International Conference on Intelligent Transportation Systems (ITSC). IEEE, Nov. 2018. DOI: $10.1109 /$ itsc. 2018.8569938.

[10] Michael J. Seitz and Gerta Köster. "Natural discretization of pedestrian movement in continuous space". In: Physical Review E 86.4 (2012), p. 046108. DOI: 10.1103/PhysRevE. 86.046108 .

[11] Christoph Sommer, Reinhard German, and Falco Dressler. "Bidirectionally Coupled Network and Road Traffic Simulation for Improved IVC Analysis". In: IEEE Transactions on Mobile Computing 10.1 (Jan. 2011), pp. 3-15. DOI: 10.1109/tmc.2010.133.

[12] Axel Wegener et al. "TraCI: an interface for coupling road traffic and network simulators". In: Proceedings of the 11th communications and networking simulation symposium on CNS 08. ACM Press, 2008, pp. 155-163. ISBN: 1-56555-318-7. DOI: 10.1145/1400713. 1400740.

[13] Stephen Wolfram. "Statistical mechanics of cellular automata". In: Review of Modern Physics 55 (3 1983), pp. 601-644. DOI: 10.1103/RevModPhys.55.601. 